\title{
Importance of Nanotechnology in Drug Delivery
}

\author{
Nahar L* and Sarker SD \\ Medicinal Chemistry and Natural Products Research Group, School of Pharmacy and \\ Biomolecular Sciences, Liverpool John Moores University, James Parsons Building, \\ Byrom Street, Liverpool L3 3AF, United Kingdom
}

\section{Editorial}

Volume 1 Issue 5

Received Date: August 21, 2017

Published Date: August 26, 2017

*Corresponding author: Lutfun Nahar, Medicinal Chemistry and Natural Products Research Group, School of Pharmacy and Biomolecular Sciences, Liverpool John Moores University, James Parsons Building, Byrom Street, Liverpool L3 3AF, United Kingdom, Tel: 0151231 2622; E-mail: L.Nahar@ljmu.ac.uk \& drnahar@live.co.uk

\section{Editorial}

The branch of technology that deals with dimensions and tolerances of 1-100 nanometres, especially the manipulation of matter on an atomic, molecular and supramolecular scale, is known as 'Nanotechnology'. In 1959, the concepts of nanotechnology were first put forward by Richard Feynman, but the actual term 'Nanotechnology' was first introduced by Norio Taniguchi in 1974. Since its introduction, nanotechnology has played an important role in advancing almost all areas of science, particularly, drug formulation and delivery. In fact, the area of drug formulation and delivery, where nanotechnology is applied, is now known as 'Nanopharmaceutics'. As nanoparticles are accepted by cells more efficiently than larger micromolecules, they can be used as effective transport and delivery systems. For medicinal purposes, drugs can either be integrated in the matrix of the particle or attached to the particle surface.

Nanoparticles can increase solubility and stability of various drug molecules, enhance their absorption, increase permeation and retention in target tissues, improve bioavailability, protect them from premature degradation in the body, exhibit high differential uptake efficiency in the target cells over normal cells and prolong their circulation time [1]. Commonly used biocompatible and biodegradable nanoparticles in drug delivery research include nanoliposomes, nanoemulsions, lipid nanocarries, micelles and poly (lactic-co-glycolic acid) (PLGA) nanoparticles. In addition to organic nanoparticles as mentioned above, inorganic nanoparticles, e.g., gold, silver, zinc, copper oxide, aluminium oxide, iron oxide, ceramics and carbon nanoparticles are also used in drug delivery.
Many conventional drugs have limitations like poor solubility in aqueous media, poor bioavailability, poor stability and toxicity [2-4]. In fact, about $95 \%$ of all new potential therapeutics has poor pharmacokinetics and biopharmaceutical properties [5]. Therefore, there is need for developing drug delivery systems that convey the therapeutically active molecules only to the site of action, without affecting other organs and tissues. Nanotechnology now offers the technology that is necessary for overcoming these problems and in delivering drugs to a certain target with greater efficiency, utilizing the techniques like, nanoformulation and encapsulation [6]. For this purpose, nanoparticles can be designed, prepared in different shapes, sizes, compositions, and functionalized and modified physicochemically to achieve specific properties depending on the characteristics of both the drug molecule and the targeted organ.

Nanoparticles can be used in targeted drug delivery (Figure 1) at the site of disease to improve the uptake of poorly soluble drugs. They can also be applied for targeting of drugs to a specific site, and improving drug bioavailability. Arguably, the most popular use of nanotechnology in drug delivery under investigation is the use of nanoparticles to deliver drugs to cancer cells. Particles are engineered in a way that they are attracted to diseased cells, which allows direct treatment of those cells but significantly reduces damage to healthy cells in the body. Several anticancer drugs including paclitaxel, doxorubicin, 5-fluorouracil and dexamethasone have been successfully formulated using nanomaterials $[6,7]$. The development and optimization of drug delivery 


\section{Open Access Journal of Pharmaceutical Research}

approaches based in nanoparticles involves the early detection of cancer cells and/or specific tumour biomarkers, and the enhancement of the efficacy of the treatments applied. However, the application of nanotechnology is not only restricted to cancer drug delivery, but also embraces many other drugs that have poor biopharmaceutical properties.

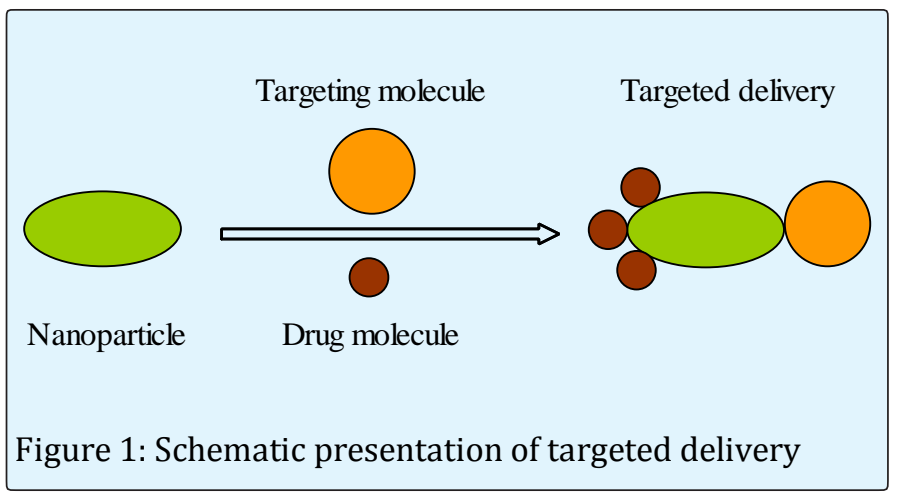

Nanotechnology has already changed, among many other fields, the field of drug delivery, influencing the enhanced and efficient delivery of various poorly bioavailable conventional drugs. There are numerous publications available to date on the use of nanoparticles for the delivery of various drug molecules, but a review by Wanigasekara and Witharana has captured the recent developments as well as the historical development of the field of nanotechnology, especially focussing on its applications drug delivery and design [8]. There is no doubt that nanoparticles have tremendous potential as an effective drug delivery system, and nanotechnology will continue to influence drug delivery research, and will facilitate the process of improving efficacy and reducing toxicity of drugs in the years to come.

\section{References}

1. Wang S, Su R, Nie S, Sun M, Zhang J, et al. (2014) Application of nanotechnology in improving bioavailability and bioactivity of diet-derived phytochemicals. J Nutr Biochem 25(4): 363-376.

2. Ansari SH, Islam F, Sameem M (2012) Influence of nanotechnology on herbal drugs: a review. J Adv Pharm Technol Res 3(3): 142-146.

3. Odeh F, Al Jaber H, Khater D (2014) Nanoflora-how nanotechnology enhanced the use of active phytochemicals. In 'Application of Nanotechnology in Drug Delivery.

4. Subramanian AP, Jaganathan SK, Manikandan A, Pandiaraj KN, Gomathi N, et al. (2016) Recent trends in nano-based drug delivery systems for efficient delivery of phytochemicals in chemotherapy. RSC Advances 6: 48294-48314.

5. Ruggiero C, Pastorino L, Herrera OL (2010) Nanotechnology based targeted drug delivery. Conf Proc IEEE Eng Med Biol Soc 3731-3732.

6. Suri SS, Fenniri H, Singh B (2007) Nanotechnologybased drug delivery systems. J Occup Med Toxicol 2: 16.

7. Piktel E, Niemirowicz K, Wątek M, Wollny T, Deptula $P$, et al. (2016) Recent insights in nanotechnologybased drugs and formulations designed for anticancer therapy. J Nanotechnology 14(1): 39.

8. Wanigasekara J, Witharana C (2016) Applications of nanotechnology in drug delivery and design - an insight. Current Trends in Biotechnology and Pharmacy 10(1): 78-91. 